\title{
Komplexer Zusammenhang
}

\section{Politik und Verbände setzen erhebliche Hoffnungen auf die Einführung von Umweltmanagementsystemen: Sie sollen die Umwelt entlasten und gleichzeitig den betrieblichen Nutzen erhöhen. Ein Schlüssel dazu sind Innovationen; die Innovationswirkungen solcher Systeme wurde bislang aber kaum untersucht. Erste Hypothesen und Ergebnisse werden im folgenden vorgestellt.}

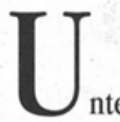
Von Heinz Kottmann,
Esther Hoffmann und Jan Nill
hmen sehen im Umweltschutz schon seit langem nicht mehr nur einen Kostentreiber, sondern betrachten ihn zunehmend von der Nutzenseite. Dabei reichen die Nutzenaspekte von der Schaffung neuer Arbeitsplätze bis hin zum Produktmarketing, von der Qualifizierung und Motivation der Mitarbeiterinnen und Mitarbeiter bis hin zur Reorganisation von Unternehmen und der Bildung von Unternehmensnetzwerken.

Mittlerweile wurden im Rahmen des Revisionsprozesses der EG-Öko-Audit-Verordnung (im folgenden EMAS, environmental management and audit scheme genannt) in einigen Studien die Auswirkungen der Verordnung untersucht. Dabei wurde allerdings der Aspekt der hierdurch ausgelösten Innovationen weitgehend ausgeklammert. In den Untersuchungen zum Thema Umweltschutz und Innovation hingegen wird zwar der Beitrag verschiedener umweltpolitischer Instrumente untersucht, betriebliche Instrumente bleiben dabei jedoch bisher oft außen vor (1).

\section{- Vorläufige Hypothesen}

Nähert man sich der Fragestellung, welche innovativen Wirkungen von Umweltmanagementsystemen ausgehen, ist es sinnvoll, sich auf Umweltmanagementsysteme nach EMAS zu konzentrieren. EMAS weist - zumindest in Deutschland - einen hohen Durchdringungsgrad auf und die Einführung von Umweltmanagementsystemen fällt häufig mit der Entscheidung zur Teilnahme an EMAS zusammen.

Aus den bisherigen Evaluationsstudien $\mathrm{zu}$ den Wirkungen von EMAS lassen sich erste Anhaltspunkte für Innovationswirkungen gewinnen. Eine Studie zur fachwissenschaftlichen Begleitung des Öko-Audit in Hessen kommt zu dem Ergebnis, dass die organisatorischen Verbesse- rungen und die positiven Wirkungen auf das operative Management zum Hauptnutzen der EMAS-Teilnahme gehören (2). Nach einer Studie von Heinelt und Töller erzielen diejenigen Unternehmen, die nicht aufgrund von externem Zwang, sondern aus interner Motivation Umweltmanagementsysteme einführen, die größeren Wirkungen (3). In diesen Unternehmen ist die Sichtweise langzeitorientiert und stellt auf weiche Faktoren, wie die Arbeitsatmosphäre und die Motivation, als Basis für Innovation und Wettbewerb ab.

Das Evaluationsprojekt zu EMAS im Auftrag des Umweltbundesamtes stellt fest, dass in den Unternehmen bislang vor allem einfach erreichbare ökologische und ökonomische Win-WinSituationen ausgeschöpft wurden (4). Weder die Reduktion der Umweltwirkungen noch die Entwicklung von neuen umwelffreundlicheren Produkten stehen bislang im Fokus der Unternehmen. Doch bietet die Fokussierung auf den Verbesserungsprozess eine große Chance, auch weiterreichende ökologische und ökonomische Veränderungen zu erreichen.

Ein von der Arbeitsgemeinschaft selbständiger Unternehmer seit 1988 regelmäßig durchgeführtes Benchmarking offenbarte insbesondere für die Jahre 1994 und 1996, dass die Unternehmen ihre Umweltmanagementsysteme im Laufe der Jahre verbessern konnten (5). Unternehmen konzentrieren sich in den ersten Jahren auf die Erfuillung der formalen Anforderungen insbesondere von EMAS oder ISO 14001. Erst Unternehmen, die schon seit längerem Umweltmanagementsysteme eingeführt haben, erzielen auch Verbesserungen in ihren Ergebnissen und Produkten (6).

Eine Analyse von Loew und Fichter zeigt schließlich auf, dass die Umweltberichterstattung sich sowohl in der Quantität wie auch in der Qualität erheblich gesteigert hat. Als Trend läßt sich die zielgruppenorientierte Ausdifferenzierung der
Umweltberichterstattung feststellen, wobei auch kommerzielle Nutzer Umweltberichte zunehmend in die Unternehmensbewertung einbeziehen (7).

Ausgehend von diesen Ergebnissen lassen sich folgende Hypothesen aufstellen:

1. EMAS wirkt vor allem indirekt und erhöht das Innovationspotenzial von Unternehmen.

2. Die Innovationswirkungen sind abhängig von der Unternehmensgröße, dem Stand der Organisationsentwicklung sowie der Umweltrelevanz der Branche.

3. Die Reichweite der eingeführten Umweltmanagementsysteme nimmt im Laufe der Zeit zu. 4. Die von EMAS vorgeschriebene Umweltberichterstattung fördert die Öffnung der Unternehmen und bahnt den Weg für innovative unternehmensübergreifende Kooperationen.

\section{Erste empirische Ergebnisse}

Im Rahmen einer groß angelegten Studie für die Generaldirektion „Industrie“ der EU-Kommission (DG III) unter Leitung des JRC-IPTS der Europäischen Kommission (Joint Research Centre, Institute for Prospective Technological Studies) in Sevilla wurden in drei Teilstudien die Innovationswirkungen von EMAS in den Ländern Italien, Großbritannien und Deutschland untersucht (8).

Einigen der Hypothesen konnte im Rahmen der deutschen Teilstudie mithilfe von fragebogengestïtzten Interviews mit ca. 20 Unternehmen der ersten EMAS-Stunden und Gesprächen mit einzelnen Verfahrensbeteiligten nachgegangen werden. Die Ergebnisse lassen sich in drei Aussagen zuspitzen:

1. EMAS führt vor allem zu positiven Änderungen in der Organisation.

2. EMAS unterstützt Innovationen direkt und indirekt.

3. EMAS führt tendenziell zu einer Erweiterung der standortbezogenen Umweltmanagementsysteme.

Fast alle befragten Unternehmen sehen in der Teilnahme am EMAS-System ein positives Kosten-Nutzen-Verhältnis, insbesondere aufgrund der organisatorischen Verbesserungen, der gestiegenen Motivation der Mitarbeiterinnen und Mitarbeiter sowie der Rechtssicherheit.

In fast allen Unternehmen hatte die Einführung von Umweltmanagementsystemen einen positiven Einfluss auf das Innovationspotenzial. Dieser Einfluss wurde über verschiedene Wege erreicht: In vielen Unternehmen wurden Pro- 
jekt- oder Innovationsteams gegründet oder Verbesserungsvorschlagssysteme um ökologische Komponenten erweitert oder neu eingeführt. Insgesamt wurden Umweltschutz und Verbesserungsmöglichkeiten in den Unternehmen häufiger diskutiert. Die generelle Einstellung zum Umweltschutz und die interne Kommunikation wurden erheblich verbessert, Lernprozesse wurden verstetigt. Insbesondere in mittleren Unternehmen führte die EMAS-Einführung zur Restrukturierung der organisatorischen Prozesse hin zu einer übergreifenden Systematik.

Auch im technologischen Bereich wurden vielfältige Innovationen durchgeführt. Hierbei sind sowohl additive als auch integrierte Techniken zu nennen, wobei integrierte Prozessinnovationen eine dominante Rolle spielen. Vielfach standen die technologischen Innovationen in Zusammenhang mit organisatorischen Veränderungen. Zwar konnten einige Unternehmen auch im Produktbereich Innovationen erzielen, doch werden Produktinnovationen im Rahmen der Umweltmanagementsysteme nicht systematisch behandelt. Gleiches gilt für externe Kooperationen: In diesem Bereich weisen zwar fast alle Unternehmen Aktivitäten auf, doch sind diese eher als ein erster Schritt zu werten.

Die Ergebnisse dieser Untersuchung stehen allerdings unter dem Vorbehalt, dass lediglich eine kleine Gruppe von Unternehmen befragt wurde und dass es keine Vergleichsgruppe gab.

\section{Ein Phasenmodell}

Aus den Analysen lässt sich ein Fortschrittsmodell zum zeitlichen Verlauf von Umweltmanagementsystemen nach EMAS ableiten:

- Phase 1: Einführung der formalen Elemente von Umweltmanagementsystemen nach EMAS;

- Phase 2: Analyse und Reorganisation der technischen und organisatorischen Prozesse;

- Phase 3: Ausweitung des standortbezogenen Umweltmanagementsystems auf Kooperationen und

- Phase 4: Übernahme der Verantwortung für den Lebenszyklus der produzierten Produkte.

In der Praxis lassen sich die vier Phasen natïrlich nicht immer so strikt trennen; vielmehr laufen verschiedene Aspekte parallel, in Abhängigkeit von Gesetzen, Markterfordernissen und personellen Schwerpunktsetzungen. So verlangen das Kreislaufwirtschaftsgesetz und die weiterführenden Regelungen für gewisse Produkte eine Produktrücknahme, so dass die hiervon betroffenen Industrien die vierte Phase bereits vorziehen mussten.
Die dritte Phase ist eine logische Konsequenz aus der bisherigen Sichtweise - der Ausschöpfung leicht erzielbarer Win-Win-Situationen -, denn in der Analyse von Wertschöpfungsketten liegt ein erhebliches Potenzial. Das Konzept des Stoffstrommanagements bietet hierfür einen instrumentellen Rahmen, der sich am Umweltcontrolling-Konzept anlehnt (9).

Eine ökologische Orientierung der Produktentwicklung als Folge von EMAS ist bislang kaum zu erkennen. Dies scheitert in Unternehmen häufig an unterschiedlichen Zuständigkeiten: Auf der einen Seite ist der Umweltmanagementbeauftragte zuständig für das Umweltmanagement und die ökologische Ausrichtung der Produktion und auf der anderen Seite die F\&E-Abteilung für die Produktentwicklung, die relativ unbeeinflusst von EMAS bleibt. Selbst auf der internationalen Normungsebene ist erkennbar, dass produktbezogene und betriebsbezogene Normungsaktivitäten in der ISO 14000er Normenreihe von unterschiedlichen Personenkreisen durchgefïhrt werden.

\section{- Ausblick}

Die Revisionsentwürfe der EMAS-Verordnung sehen die Aufnahme der ISO 14001 als Basis für die EMAS-Validierung vor. Hieraus wird sich demnächst in Deutschland viel stärker die Frage stellen, inwieweit ein Unternehmen zusätzlich zum ISO-Standard eine Registrierung benötigt. Der zusätzliche externe Nutzen von EMAS bekommt damit eine entscheidende Bedeutung. In diesem Zusammenhang ist die Klärung der Frage interessant, ob EMAS stärkere Innovationswirkungen als ISO 14001 hervorruft. Eine Hypothese ist, dass die besondere Orientierung von EMAS auf die kontinuierliche Verbesserung der Umweltleistung verbunden mit dem zusätzlichen Druck der Umweltberichterstattung einen Unterschied ausmacht. Eine wichtige Forschungsfrage ist daher, welche Bestandteile von Umweltmanagementsystemen die größten Innovationswirkungen hervorrufen.

Innovationen erstrecken sich über einen längeren Zeitraum, weshalb Längsschnittanalysen ein geeigneter Untersuchungsweg sind. Die wenigen Längsschnittuntersuchungen zu EMAS widmen sich den Innovationswirkungen bisher nur unzureichend. Nur im Längsschnitt lässt sich die dem Phasenmodell zugrundeliegende Hypothese, dass die entscheidenden Innovationswirkungen vom kontinuierlichen Verbesserungsprozess und nicht von der erstmaligen Einrichtung des
Managementsystems ausgehen, erhärten. Ähnliches gilt für die Hypothese der zunehmenden Reichweite.

Nicht zuletzt könnten anhand der Analyse der Innovationswirkungen von Umweltmanagementsystemen auch Lücken der bisherigen Forschung zu Umwelt und Innovation geschlossen werden. Gerade der hier besonders relevante Bereich von organisatorischen Innovationen und seine Wechselwirkungen mit technologischen Innovationen sind noch kaum erforscht oder operationalisiert.

\section{Anmerkungen}

(1) Vgl. z. B. Klemmer, P./ U. Lehr/ K. Löbbe: Umweltinnovationen - Anreize und Hemmnisse, Berlin 1999 und Klemmer, P. (Hrsg.): Umwelt und Innovation, Fallstudien zum Anpassungsverhalten in Wirtschaft und Gesellschaft, Berlin 1999 sowie für einen Überblick den Schwerpunkt von Ökologisches Wirtschaften 2/99.

(2) Hessisches Ministerium für Umwelt, Energie, Jugend, Familie und Gesundheit (Hrsg.): Fachwissenschoftliche Bewertung des EMAS-Systems (Öko-Audit) in Hessen, Endbericht zum Forschungsvorhaben, August 1998.

(3) Heinelt, H. / A.E. Töller: Vom Fremdzwang zum Selbstzwang, Zur Praxis von Öko-Audits in Deutschland, in Zeitschrift für Rechtssoziologie 1/99, S. 82-104.

(4) Umweltbundesamt (Hrsg.): Umweltmanagement in der Praxis, Teilergebnisse zur Vorbereitung der 1998 vorgesehenen Überprüfung des gemeinschaftlichen Öko-AuditSystems. UBA-Texte 20/98 (Teile I bis III) und 52/98 (Teile IV bis VI), Berlin 1998.

(5) Unternehmerinstitut e.V. und Arbeitsgemeinschaft Selbständiger Unternehmer: Öko-Audit in der mittelständischen Praxis, Bonn 1997.

(6) Clausen, J.: Monitoring von Umweltleistung und Umweltmanagementsystemen, Diskussionspapier des IÖW 43/98, Berlin 1998.

(7) Loew, T./ K. Fichter: Umweltberichterstattung in Deutschland und Europa. IÖW-Schriftenreihe Nr. 138/99, Berlin 1999.

(8) Vgl. dazu Hemmelskamp, J./ F. Leone: The Impact of Regulation on Innovation in European Industry und Kottmann, H.: EMAS und Innovation, in Ökologisches Wirtschaften $2 / 99$, S. 20.

(9) Vgl. z.B. Umweltbundesamt (Hrsg.): Aufgaben des betrieblichen und betriebsübergreifenden Stoffstrommanagements, UBA Texte 11/97, Berlin 1997.

\section{Die Autorinnen}

Heinz Kottmann ist freier wiss. Mitarbeiter, Esther Hoffmann und Jan Nill sind wiss. MitarbeiterInnen am IÖW.

Kontakt: I0̈W, Potsdamer Str. 105, 10785

Berlin. Tel. 030/884594-0, Fax 030/8825439, E-mail: Esther.Hoffmann@ioew.de 
(c) 20I0 Authors; licensee IÖW and oekom verlag. This is an article distributed under the terms of the Creative Commons Attribution Non-Commercial No Derivates License (http://creativecommons.org/licenses/by-nc-nd/3.o/), which permits unrestricted use, distribution, and reproduction in any medium, provided the original work is properly cited. 